\title{
Aberrant Insertion of the Right Subclavian Artery: an Unusual Cause of Dysphagia in an Adult
}

\author{
Arjan P. Schouten van der Velden • Paul Berger • \\ Attila G. Krasznai • Peter van Duijvendijk • \\ J. Adam van der Vliet
}

Received: 19 May 2009 / Accepted: 3 June 2009 / Published online: 26 June 2009

(C) The Author(s) 2009. This article is published with open access at Springerlink.com

\begin{abstract}
Introduction Within this report, we present a patient with difficulty of swallowing caused by an aberrant right subclavian artery.

Discussion This is a congenital anomaly with the right subclavian artery originating from the dorsal part of the aortic arch and coursing through the mediastinum between the esophagus and the vertebral column. The diagnosis and treatment of this disorder is discussed based on the findings from the literature.
\end{abstract}

Keywords Dysphagia · Lusoria · Esophagus .

Vascular anomaly $\cdot$ Subclavian

\section{Introduction}

Usual causes of difficulty of swallowing or dysphagia in adults include malignancy, esophageal motility disorders, or esophageal strictures. An uncommon cause of dysphagia is compression of the esophagus by a vascular structure. The clinical syndrome of dysphagia in association with an aberrant right subclavian artery compressing the esophagus was first discovered by David Bayford (1739-1790) and reported as dysphagia lusoria after "lusus naturea" (freak of nature) in $1787 .{ }^{1}$ An aberrant right subclavian artery is the most common embryologic abnormality of the aortic arch and occurs in $0.5 \%$ to $1.8 \%$ of the population, but it is usually asymptomatic. ${ }^{2,3}$ Within this report, we present a patient with dysphagia lusoria and discuss the anatomical abnormality, diagnosis, and treatment of this rare entity.

A. P. Schouten van der Velden $(\bowtie) \cdot$ P. Berger $(\bowtie) \cdot$

A. G. Krasznai · P. van Duijvendijk · J. A. van der Vliet

Department of Surgery, Division of Vascular Surgery,

Radboud University Nijmegen Medical Centre,

PO Box 9101, 65oo HB Nijmegen, The Netherlands

e-mail: aschoutenvandervelden@hotmail.com

e-mail: p.berger@chir.umcn.nl

\section{Case Report}

A 74-year-old woman was referred to our university hospital because of intermittent dysphagia with difficulty swallowing solid foods. Her medical history included a breast carcinoma for which she underwent breast-conserving treatment 3 years earlier. She had been put on proton pump inhibitors since several years without relieving her symptoms. At physical examination, no abnormalities were found. By report from the referring hospital, an endoscopy revealed no abnormalities besides a hiatal hernia without the aspect of a Barrett's esophagus or gastric inflammation. A barium contrast examination demonstrated a compression of the proximal esophagus at the level of the aortic arch suggesting of a mass compressing the esophagus (Fig. 1). Therefore, a computed tomography (CT) angiography scan was performed, which revealed an aberrant right subclavian artery arising from the aortic arch causing compression of the esophagus (Fig. 2). Because of the severity of symptoms and the inability to eat had caused weight loss, surgical treatment was indicated.

Under general anesthesia, she was placed in a half supine position and via a right supraclavicular approach, the right carotid and vertebral artery were identified. The aberrant right subclavian artery was dissected free from the esophagus and mobilized into the mediastinum. After administration of $5,000 \mathrm{IU}$ of heparin intravenously, the proximal right subclavian artery was transected with an endostapler (Multifire Endo TA 30, Covidien, MA, USA) and cut. The distal 
Figure 1 A barium contrast examination revealing a filling defect at the level of the aortic arch. In the posterior-anterior view, the parts of the esophagus proximal and distal to the defect are displaced to each other (arrow, a). In the lateral projection, a wedge-shaped impression on the dorsal esophagus is seen (arrow, b).
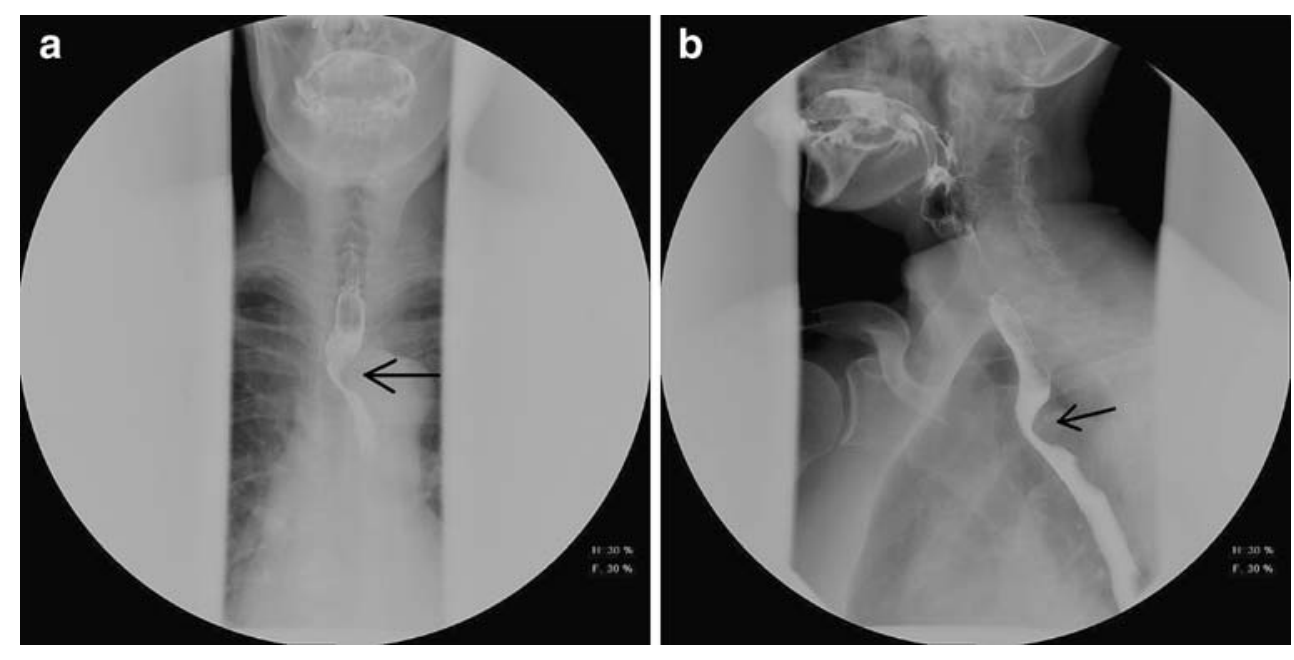

artery was anastomosed end to side to the right aortic artery (Figs. 3, 4, and 5). Immediately after the operation, the dysphagia had disappeared. The patient uneventfully recovered and was discharged 2 days after surgery. At 6 weeks of follow-up, a CT angiography showed a patent arterial reconstruction (Fig. 6).

\section{Discussion}

During early embryologic development, the aortic arches start as a duplicate system. The right aortic arch disappears proximally to form the right subclavian and common carotid artery. These latter vessels fuse to form the brachiocephalic trunk (or innominate artery) which is usually the first branch of the aortic arch. Abnormal involution of the right aortic arch with a persisting (seventh) intersegmental artery results in the

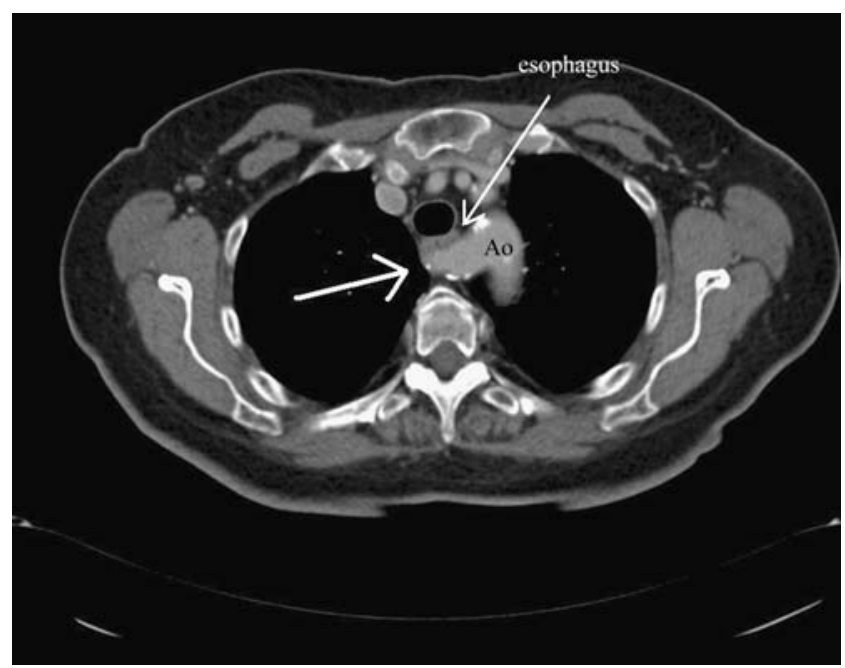

Figure 2 A CT angiography which shows the aberrant origin of the right subclavian artery (white arrow). Note the close relationship with the esophagus. Ao aortic arch. evolution of an aberrant right subclavian artery. ${ }^{3}$ The aberrant right subclavian artery arises from the dorsal part of the aortic arch with a broad base formed by a remnant of the persisting primitive right aorta, the so-called Kommerell's diverticulum. ${ }^{4}$ The aberrant subclavian or lusoria artery passes through the mediastinum between the esophagus and the vertebral column to reach the right axilla in the majority of cases. ${ }^{5,6}$ An aberrant right subclavian artery is in approximately one third of cases associated with carotid artery anomalies with a common origin of the left and right carotid artery (bicarotid truncus). ${ }^{6}$

The majority of patients with an aberrant right subclavian artery will remain asymptomatic during lifetime. ${ }^{2,3,6}$ In symptomatic infants, it usually presents with respiratory signs. This is most likely due to the absence of tracheal rigidity, allowing its compression to lead to airway obstruction with recurrent pulmonary infection. ${ }^{5-7}$ It is not clear

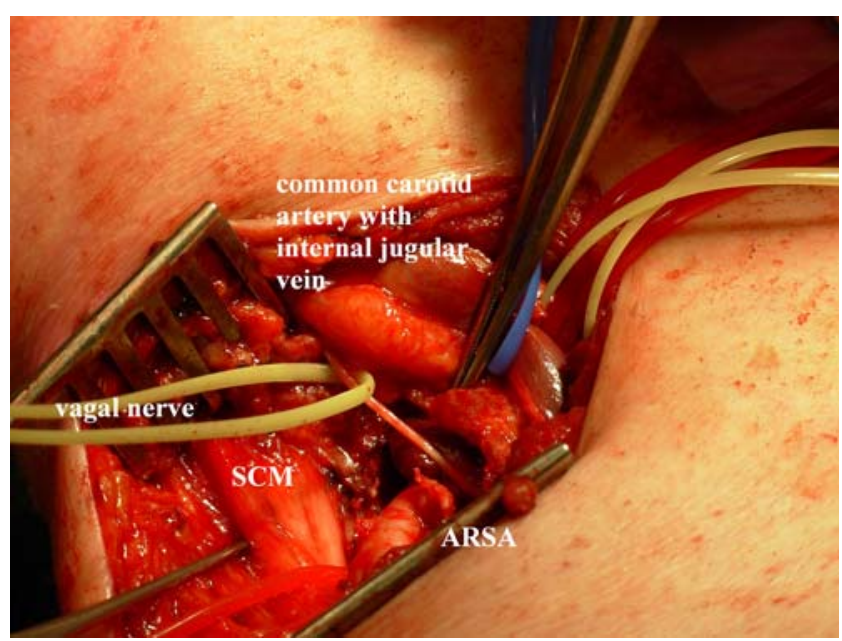

Figure 3 An intraoperative view showing the aberrant right subclavian artery crossing dorsally to the common right carotid artery. SCM sternocleidomastoid muscle. 


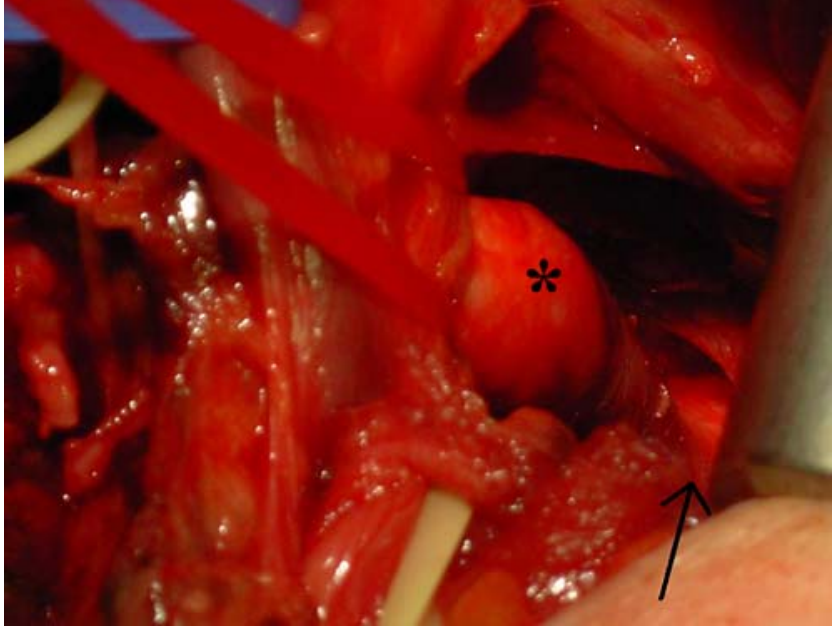

Figure 4 An intraoperative view showing the aberrant right subclavian artery (asterisk) crossing dorsally to the esophagus (arrow).

why dysphagia occurs in older patients. It could be attributed to increasing rigidity of the esophagus or arterial elongation and thickening due to atherosclerosis during lifetime. ${ }^{7}$ Similarly, it is uncertain whether dysphagia occurs primarily due to esophageal impression or secondary to motility changes. $^{7}$ Others hypothesized that the coincidence of a common carotid origin and an aberrant right subclavian artery can give rise to compression of the esophagus between these vessels. ${ }^{6,7}$

In adults with dysphagia, an upper gastrointestinal endoscopy is usually performed. In case of an aberrant right subclavian artery, a pulsating impression can sometimes be seen, but it usually is normal. ${ }^{5,7} \mathrm{~A}$ barium contrast examination will show a filling defect at the level

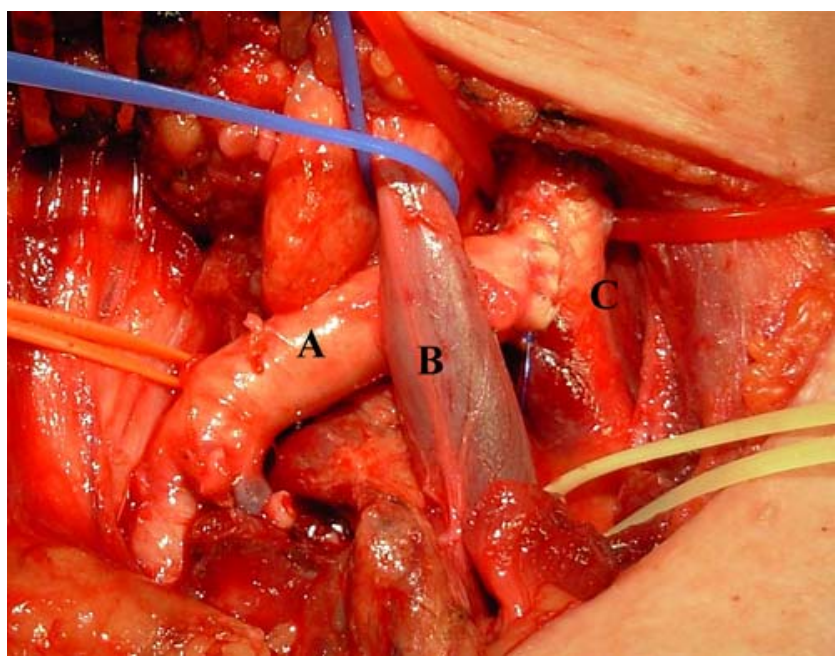

Figure 5 An intraoperative view showing the end-to-side anastomosis between the right subclavian artery $(A)$ and the right common carotid artery $(C)$. $B$ Internal jugular vein.

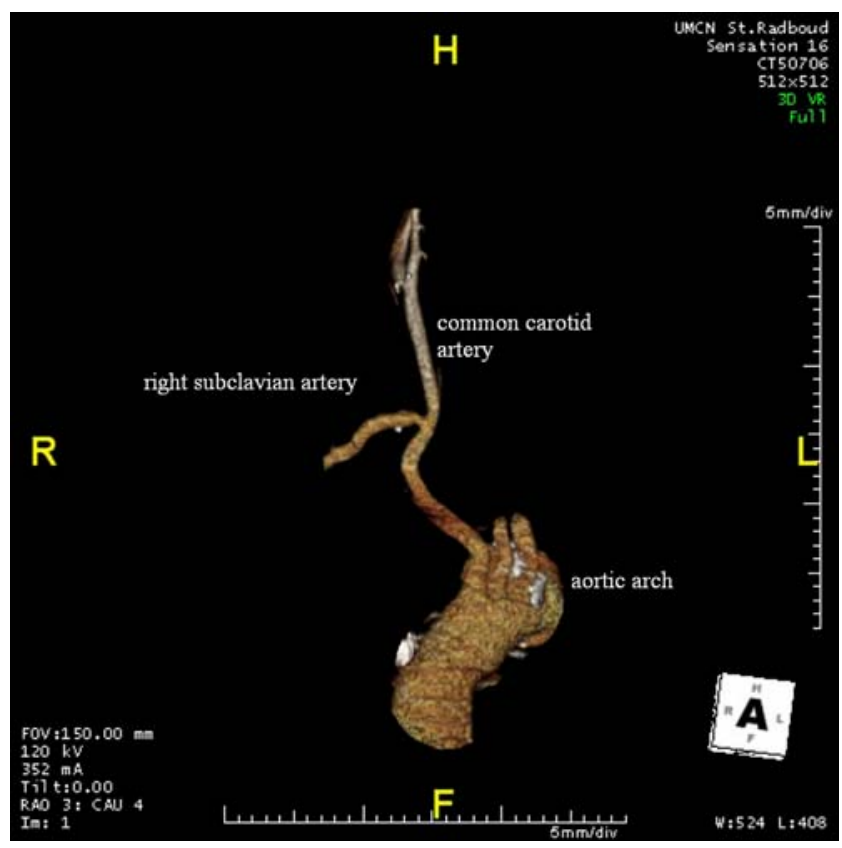

Figure 6 A $3 \mathrm{D}$ reconstruction of a postoperatively performed CT angiography showing the end-to-side anastomosis between the proximal right subclavian artery and the right common carotid artery.

of the aortic arch, as illustrated in our patient (Fig. 1). In the posterior-anterior view, the parts of the esophagus proximal and distal from the defect appear displaced (Fig. 1a). ${ }^{6}$ In the lateral projection, a wedge-shaped impression on the dorsal esophagus is seen (Fig. 1b). ${ }^{6}$ CT or magnetic resonance (MR) angiography is considered as the gold standard for the diagnosis of an aberrant right subclavian artery. ${ }^{8}$

The management of these patients depends on the severity of their symptoms. Janssen and colleagues reported three out of six patients with dysphagia lusoria who became free of symptoms after dietary changes or acid inhibition or promotility agents. ${ }^{7}$ In case of severe or persistent symptoms, surgical intervention is warranted to remove the aberrant vessel and reconstruct the vascular supply. The surgical approach depends on the vascular anatomy: An isolated supraclavicular approach can generally be used if the aortic arch is normal without aneurysm formation of the proximal aberrant right subclavian artery, as in the presented patient. In patients with associated lesions, a combined cervical and thoracic approach might be more appropriate. ${ }^{9}$ Simple ligation and division of the aberrant subclavian artery is likely to cause ischemia of the upper limb or a "subclavian steal" syndrome; therefore, subclavian carotid transposition is advocated (reimplantation of the right subclavian artery to the right common carotid artery), as we used in our patient. ${ }^{10}$ The results of surgical treatment are excellent with relieve of symptoms in nearly all reported patients. ${ }^{7,9}$ 
In conclusion, dysphagia in an adult patient can be attributed to an anomaly of the right subclavian artery and this should be included in the differential diagnosis. A barium swallow examination can give a clue toward the diagnosis, but CT or MR imaging angiography is the golden standard for diagnosing this anomaly. In cases with severe symptoms resistant to medical therapy, surgical intervention should be considered. If no aneurysm or aortic disease is present, a supraclavicular approach with diversion of the proximal aberrant artery and an end-to-side anastomosis of the distal right subclavian artery to the right common carotid artery are recommended.

Conflicts of Interests For this manuscript, there was no financial support nor are there any conflicts of interests.

Open Access This article is distributed under the terms of the Creative Commons Attribution Noncommercial License which permits any noncommercial use, distribution, and reproduction in any medium, provided the original author(s) and source are credited.

\section{References}

1. Asherson N, Bayford D. His syndrome and sign of dysphagia lusoria. Ann R Coll Surg Engl 1979;61:63-67.

2. Carrizo GJ, Marjani MA. Dysphagia lusoria caused by an aberrant right subclavian artery. Tex Heart Inst J 2004;31:168-171.

3. Molz G, Burri B. Aberrant subclavian artery (arteria lusoria): sex differences in the prevalence of various forms of the malformation. Virchows Arch A Pathol Anat Histopathol 1978;380:303-315.

4. Van Son JAM, Konstantinov IE, Burckhard F. Kommerell and Kommerell's diverticulum. Tex Heart Inst J 2002;29:109-112.

5. Levitt B, Richter JE. Dysphagia lusoria: a comprehensive review. Dis Esophagus 2007;20:455-460.

6. Klinkhamer AC. Aberrant right subclavian artery. Clinical and radiological aspects. Am J Roentgenol 1966;97:438-436.

7. Janssen M, Baggen MGA, Veen HF, Smout AJPM, Bekkers JA, Jonkman JGJ, Ouwendijk RJTh. Dysphagia lusoria: clinical aspects, manometric findings, diagnosis, and therapy. Am J Gastroenterol 2000;95:1411-1416.

8. Karcaaltincaba M, Haliloglu M, Ozkan E, Kocak M, Akinci D, Ariyurek M. Non-invasive imaging of aberrant right subclavian artery pathologies and aberrant right vertebral artery. Br J Radiol 2009;82:73-78.

9. Kieffer E, Bahnini A, Koskas F. Aberrant subclavian artery: surgical treatment in thirty-three adult patients. J Vasc Surg 1994;19:100-111.

10. Edwards WH, Tapper SS, Edwards WH Sr, Mulherin JL, Martin RS, Jenkins JM. Subclavian revascularisation. A quarter century experience. Ann Surg 1994;219:673-678. 